\title{
Mapeando usos/coberturas da terra com Semi-automatic Classification Plugin: quais dados, classificador e estratégia amostral?
}

\author{
Luís Flávio PEREIRA ${ }^{1 *}$, Ricardo Morato Fiúza GUIMARÃES² \\ ${ }^{1}$ Departamento de Solos, Universidade Federal de Viçosa, Viçosa, MG, Brasil. \\ ${ }^{2}$ Escola de Minas, Universidade Federal de Ouro Preto, Ouro Preto, MG, Brasil. \\ *E-mail: pereira.flavioluis@gmail.com
}

\begin{abstract}
Recebido em junho/2018; Aceito em outubro/2018.
RESUMO: Este trabalho teve como objetivo sugerir diretrizes para melhor mapear usos da terra usando 0 complemento Semi-automatic Classification Plugin (SCP) para QGIS, destacando-se quais os melhores conjuntos de dados, classificadores e estratégias amostrais para treinamento. Foram combinados quatro conjuntos de dados derivados de imagem Sentinel 2A, três classificadores disponíveis no SCP, e duas estratégias amostrais: amostras de treinamento (ROI's) separadas ou dissolvidas em uma única amostra, obtendo-se 24 tratamentos. Os tratamentos foram avaliados quanto à acurácia (coeficiente Kappa), qualidade visual do mapa final e tempo de processamento. Os resultados mostraram que: (1) o SCP é adequado para mapear usos da terra; (2) quanto maior o conjunto de dados, melhor o desempenho do classificador; e (3) a utilização de ROI's dissolvidas sempre diminui o tempo de processamento, mas apresenta efeito ambíguo sobre os diferentes classificadores. Para melhores resultados, recomenda-se a aplicação do classificador Maximum Likelihood sobre o maior conjunto de dados disponível, utilizando-se amostras de treinamento coletadas contemplando todas as variações intraclasse, e posteriormente dissolvidas em uma única ROI.
\end{abstract}

Palavras-chave: sensoriamento remoto, amostras de treinamento, QGIS, Sentinel 2A,

\section{Mapping land uses/covers with Semi-automatic Classification Plugin: which data set, classifier and sampling design?}

\begin{abstract}
This paper aimed to suggest guidelines to better map land uses using the Semi-automatic Classification Plugin (SCP) for QGIS, highlighting which the best data sets, classifiers and training sampling designs. Four data sets from a Sentinel $2 \mathrm{~A}$ image were combined with three classifiers available in the SCP, and two sampling designs: separate or dissolved training samples (ROI's) in a single sample, obtaining 24 treatments. The treatments were evaluated regarding the accuracy (Kappa coefficient), visual quality of the final map and processing time. The results suggest that: (1) the SCP is suitable to map land uses; (2) the larger the data set, the better the classifier performance; and (3) the use of dissolved ROI always decreases processing time, but has an ambiguous effect on the different classifiers. In order to get better results, we recommend to apply the Maximum Likelihood classifier on the largest data set available, using training samples that cover all possible intraclass variations, subsequently dissolved in a single ROI.
\end{abstract}

Keywords: remote sensing, training samples, QGIS, Sentinel 2A.

\section{INTRODUÇÃO}

Mudanças de uso da terra podem provocar impactos, como alterações climáticas e hidrológicas, proliferação de doenças e degradação do solo. A intensidade desses impactos depende de fatores relacionados aos meios físico, social e ambiental, e ainda do nível de mudança do uso das terras (FOLEY et al., 2005; EIDT et al., 2016; DOS SANTOS et al., 2017; PEREIRA et al, 2018a). Dessa forma, mapeamentos de uso da terra têm importância na compreensão da dinâmica agrária, fisiográfica, ecológica e sociológica de uma região. Entretanto, mapeamentos adequados para análise ambiental e planejamento agrário (escalas detalhadas, geralmente com alta especificidade categórica) muitas vezes têm sido impossibilitados por dificuldades técnicas e orçamentárias, pois podem exigir recursos dispendiosos e abordagens complexas (JENSEN, 2009). Por isso, a utilização de softwares livres, metodologias simples e/ou bases de dados gratuitas na avaliação do uso/cobertura da terra via sensoriamento remoto tem aumentado (GUIMARÃES et al., 2012; PEREIRA et al., 2018a).

Dentre os softwares livres em ascensão, destaca-se o QGIS. Esse software é um Sistema de Informações Geográficas livre e aberto, possui interface intuitiva e permite uma infinidade de funcionalidades, à medida que novos complementos e integrações com outros softwares têm sido rapidamente implementados. Essas características tornam o QGIS adaptável às necessidades de qualquer usuário (GUIMARÃES et al., 2012; NANNI et al., 2017).

Um dos mais importantes plugins do QGIS é o Semiautomatic Classification Plugin (SCP). O SCP foi desenvolvido por Luca Congedo em 2013, e vem sendo frequentemente atualizado. É um aplicativo para classificação supervisionada de imagens, e é considerado completo, por permitir desde o download de imagens até a avaliação estatística dos mapas produzidos (CONGEDO, 2016). Sua utilização é crescente e tem gerado bons resultados (LEROUX 
et al., 2018; CONGEDO, 2016), mas ainda não existem trabalhos que comparam sistematicamente estratégias para melhorar seu desempenho.

A identificação de estratégias que melhorem o desempenho do SCP, portanto, é prioritária, pois traria economia de recursos aos usuários, além de potencializar a utilização do plugin. Pesquisadores da classificação supervisionada de imagens têm se debruçado principalmente sobre a forma de amostragem, os dados espectrais utilizados e o classificador empregado, por serem variáveis que exercem forte influência sobre a qualidade dos mapas obtidos (Castillejo-González et al., 2009; Yang et al., 2008; Sameen et al., 2016; Faria, 2015).

Este trabalho teve como objetivo sugerir diretrizes para utilização do SCP, com foco sobre quais dados, classificador e forma de amostragem utilizar.

\section{MATERIAL E MÉTODOS}

Para avaliar qual a melhor configuração do SCP, foram combinados quatro conjuntos de dados, duas estratégias amostrais e os três classificadores disponíveis no SCP, o que resultou em 24 tratamentos. Foram definidas 15 classes a se mapear. Os critérios de seleção dos melhores tratamentos foram a acurácia da classificação, qualidade visual do mapa final e o tempo de processamento. As versões utilizadas do SCP e do QGIS foram 5.3.11 - Kourou e 2.18.16 - Las palmas, respectivamente. Os detalhamentos metodológicos são apresentados a seguir.

\subsection{Local de estudo e conjuntos de dados}

A área de estudo adotada é apresentada na Figura 1, e consistiu numa parcela de $830 \mathrm{~km}^{2}$ localizada no sudeste mineiro, coberta por Mata Atlântica. A área engloba parte da serra da Mantiqueira e do planalto do alto rio Doce, na Zona da Mata e Campo das Vertentes de Minas Gerais, e foi escolhida por sua grande diversidade de terrenos e coberturas da terra (além da alta variabilidade intracoberturas) (LEITE et al., 2007; PEREIRA et al., 2018a). Os dados espectrais adotados consistiram em uma imagem obtida pela plataforma Sentinel, sensor $2 \mathrm{~A}$ S2A_OPER_MSI_L1C_TL_SGS_20160424T130942_2016 0424T181611_A004385_T23KPS_N02_01_01; data de aquisição pelo sensor: $24 / 04 / 201 \overline{6}$, final da época chuvosa), obtida junto ao United States Geological Survey (USGS) já com correções radiométricas e geométricas, ortorreterificação e o registro espacial (nível 1C). O download foi realizado mediante cadastro e solicitação no site da USGS.

A imagem Sentinel 2A foi escolhida por apresentar grande resolução espectral e espacial (Tabela 1), o que permite mapeamentos multicategóricos e em escala detalhada (JENSEN, 2009). A imagem foi corrigida atmosfericamente pelo método Dark Object Subtraction (DOS1), que apesar de simples, é o único disponível no SCP, e apresenta desempenho adequado e similar ao de outros métodos mais complexos (PRIETO-AMPARAN et al., 2018). As classes mapeadas foram: Milho (lavouras maduras); Cana (lavouras maduras); Talhões Agrícolas Indiferenciados; Café; Eucalipto; Brejos; Pastagem Sem Degradação; Pastagem Moderadamente Degradada; Pastagem Severamente Degradada; Mata; Regeneração Natural (capoeiras); Rochedos e Cidades; Água; Nuvem; e Sombra. As classes foram codificadas de 1 a 15, na sequência aqui apresentada.

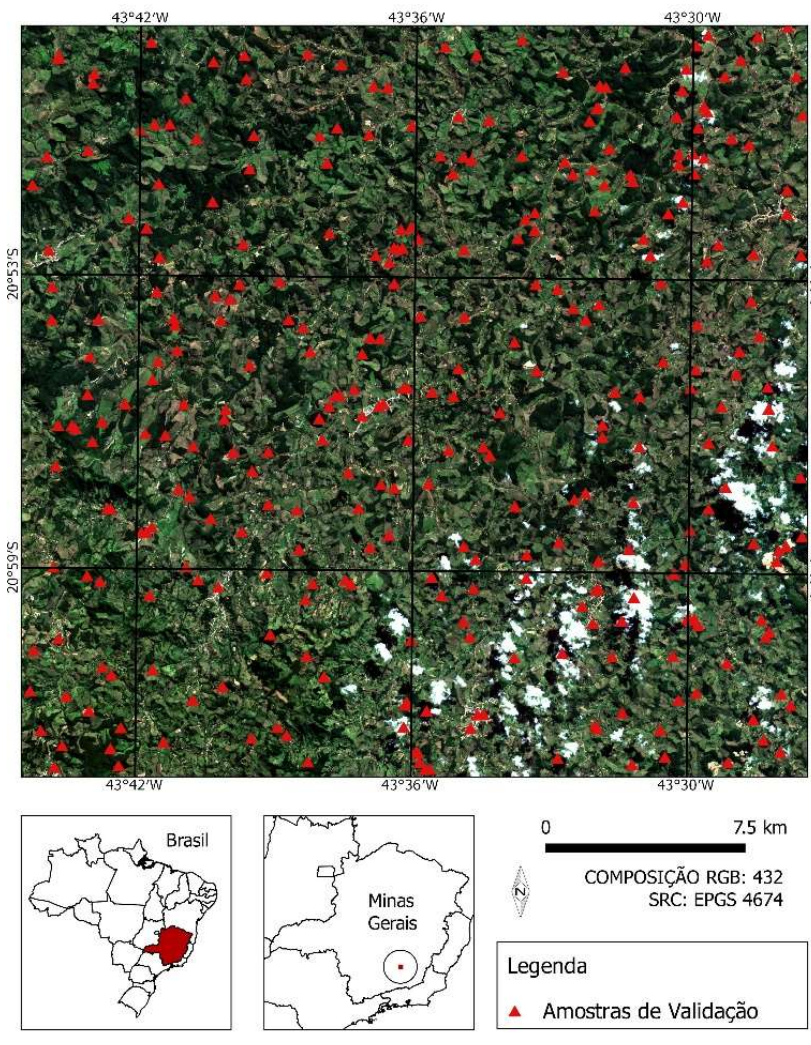

Figura 1. Localização da área de estudo e das amostras de validação. Figure 1. Location of study area and of validation samples.

Os dados espectrais disponíveis foram utilizados para o cálculo de 5 índices espectrais, que incluíram índices de vegetação, água e umidade, resultando em um total de 15 planos de informação disponíveis (bandas). Os planos de informação foram organizados em quatro conjuntos de dados, com número crescente de planos de informação do conjunto D0 ao D3, conforme apresentados na Tabela 1.

\subsection{Classificação no SCP}

O SCP opera classificações do tipo supervisionada, em que é necessário fornecer uma amostra de pixels representativos para cada classe que se deseja mapear. No SCP essa amostra de pixels é delimitada por polígonos, e recebe o nome de "Region of Interest" (ROI). Comumente, muitas ROI's para uma mesma classe devem ser coletadas, devido à necessidade de representar a variabilidade espectral intraclasse, frequentemente alta. Utilizou-se duas estratégias diferentes para coleta das ROI's: a primeira consistiu em coletar pequenos polígonos com, em média, 36 pixels, fornecendo cada polígono como uma ROI. A segunda consistiu em fornecer os mesmos polígonos, mas dessa vez dissolvidos em uma única ROI. Para emprego dessas estratégias, coletou-se as amostras em polígonos no formato shapefile, os quais foram posteriormente importados pelo SCP para o cálculo da assinatura espectral.

As amostras foram selecionadas a partir de trabalhos de campo, e por interpretação de composições RGB 432 da imagem Sentinel 2A e de modelos tridimensionais e séries temporais de imagens do Google Earth Pro, conforme o método descrito por Pereira et al. (2018a). Os níveis de degradação de pastagens foram definidos a partir da fusão de classes de degradação apresentadas para a região por Pereira et al. (2018b): Classe V = Pastagem Sem Degradação; Classes 
III e IV = Pastagem Moderadamente Degradada; e Classes I e II = Pastagem Severamente Degradada.

Tabela 1. Descrição das bandas Sentinel 2A utilizadas (DRUSCH et al., 2012), bem como índices espectrais e conjuntos de dados obtidos. Table 1. Description of the Sentinel 2A bands used (DRUSCH et al., 2012), as well as spectral indices and the data sets obtained.

\begin{tabular}{|c|c|c|c|}
\hline Banda & Nome & Pixel $(\mathrm{m})$ & $\lambda$ central $(\mathrm{nm})$ \\
\hline $\mathrm{B} 2$ & Blue & 10 & 480 \\
\hline B3 & Green & 10 & 560 \\
\hline B4 & Red & 10 & 665 \\
\hline B5 & Red Edge 1 & 20 & 705 \\
\hline B6 & Red Edge 2 & 20 & 740 \\
\hline B7 & Red Edge 3 & 20 & 783 \\
\hline B8 & NIR & 10 & 842 \\
\hline $\mathrm{B} 8 \mathrm{~A}$ & Red Edge 4 & 20 & 865 \\
\hline $\mathrm{B} 11$ & SWIR 1 & 20 & 1610 \\
\hline $\mathrm{B} 12$ & SWIR 2 & 20 & 2190 \\
\hline $\begin{array}{l}\text { Índices } \\
\text { Espectrais }\end{array}$ & Aplicação & Bandas & Autor \\
\hline EVI & Vegetação & $\mathrm{B} 2, \mathrm{~B} 4$ e $\mathrm{B} 8$ & Huete et al. (2002) \\
\hline NDVI & Vegetação & B4 e B8 & Rouse et al. (1973) \\
\hline NDWI 1 & Água & $\mathrm{B} 3$ e $\mathrm{B} 8$ & McFeeters (1996) \\
\hline NDWI 2 & Umidade & B8 e B11 & Gao (1996) \\
\hline VDVI & Vegetação & $\mathrm{B} 2, \mathrm{~B} 3$ e $\mathrm{B} 4$ & Xiaoqin et al. (2015) \\
\hline Composição & \multicolumn{2}{|c|}{ Dados utilizados } & Resolução (m) \\
\hline $\mathrm{D} 0$ & \multicolumn{2}{|c|}{ Bandas no Visível e NIR } & 10 \\
\hline $\mathrm{D} 1$ & \multicolumn{2}{|c|}{ D0 + Red Edge 1 a 4} & 10 \\
\hline $\mathrm{D} 2$ & \multicolumn{2}{|c|}{ D $1+$ SWIR 1 e 2} & 10 \\
\hline D3 & \multicolumn{2}{|c|}{ D2 + Índices Espectrais } & 10 \\
\hline
\end{tabular}

Foram coletados números distintos de pixels/ROI's para cada classe mapeada. O número de pixels foi maior quanto maiores a abrangência e variabilidade interna de cada classe de interesse: 800 para classes abundantes e altamente variáveis (Matas, Pastagens Conservadas e Moderadamente Degradadas), 600 para classes de variabilidade e ocorrência moderadas (Café, Talhões e Pastagem Severamente Degradada), e 480 pixels para as demais classes, de baixas ocorrência ou variabilidade. Considerou-se que o desbalanceamento no número de pixels/ROI's coletados não influenciou a classificação, visto que Faria (2015) mostrou que para diversos classificadores não há influência do número de pixels na amostra de treinamento sobre a acurácia final do mapa, desde que utilizados no mínimo 80 pixels divididos em pequenos polígonos. Além disso, é importante salientar que o mesmo conjunto de treinamento foi fornecido para todos os tratamentos, de modo a garantir que a amostragem de treinamento não consistisse em fonte de variação.

Os pixels são classificados um a um, de acordo com as semelhanças espectrais entre ROI's e pixels. No SCP há três classificadores disponíveis: Maximum Likelihood (ML), Minimum Distance (MD) e Spectral Angle Mapper (SAM). Os três classificadores foram utilizados, e os parâmetros para cada classificador foram mantidos como a configuração padrão do SCP.

O ML classifica os pixels como pertencentes à classe mais provável, baseando-se em funções de densidade de probabilidade derivadas da média e da covariância dos valores de pixels para cada ROI (CONGEDO, 2016). O MD se baseia na distância euclidiana mínima entre o valor de cada pixel desconhecido e a média de cada ROI para todos os planos de informação. O MD calcula a distância entre pontos em um espaço n-dimensional, em que $\mathrm{n}$ é o número de bandas utilizadas, e cada pixel é então classificado como pertencente à classe em que a distâcia entre o pixel e determinada ROI for a menor (CONGEDO, 2016). Enquanto isso, o SAM considera cada pixel e ROI como vetores no espaço n-dimendional, e o pixel é classificado como pertencente à classe do ROI em que o ângulo entre os dois vetores for o menor (CONGEDO, 2016).

\subsection{Avaliação Estatística}

Para avaliação da acurácia dos mapas, foi utilizado o índice Kappa, calculado conforme Congalton; Green (2008). O Kappa é uma métrica derivada da matriz de confusão entre dados classificados e uma amostra de referência, e é amplamente utilizado na avaliação da acurácia de produtos obtidos por sensoriamento remoto (CONGALTON; GREEN, 2008). O valor máximo para o índice Kappa é 1, quando a classificação apresenta concordância total entre dados classificados e uma amostra referência, e seus valores podem ser interpretados em classes de concordância, conforme a Tabela 2.

Tabela 2. Interpretação dos valores de Kappa (adaptado de Landis; Koch (1977)), e nível de adequabilidade aceitáveis para mapeamentos multicategóricos no presente estudo.

Table 2. Interpretation of the Kappa values (adapted from Landis; Koch (1977)), and acceptable level of adequacy for multicategorical mappings in the present study.

\begin{tabular}{llr}
\hline Kappa & Interpretação & Adequabilidade \\
\hline$<0$ & Sem concordância & Inadequado \\
0 a 0,19 & Concordância muito baixa & Inadequado \\
0,20 a 0,39 & Concordância baixa & Inadequado \\
0,40 a 0,59 & Concordância moderada & Inadequado \\
0,60 a 0,79 & Concordância alta & Adequado \\
0,80 a 1,00 & Concordância muito alta & Adequado \\
\hline
\end{tabular}

Os valores de Kappa obtidos para cada tratamento foram comparados um a um utilizando-se o teste $\mathrm{Z}$ apresentado por Congalton; Green (2008), conforme a Equação 1. Um teste Z descrito pelos mesmos autores também foi utilizado para verificar a significância de cada Kappa, separadamente.

$$
Z=\frac{\left|\hat{K}_{i}-\hat{K}_{j}\right|}{\sqrt{\operatorname{Va} r\left(\hat{K}_{i}\right)-\operatorname{Var}\left(\hat{K}_{j}\right)}}
$$

em que: $\hat{K}_{i e j}=$ Kappas a serem comparados, adimensional; e $\operatorname{Vâ} r\left(\hat{K}_{i e j}\right)=$ variâncias dos Kappas em análise.

A variância do índice Kappa é também uma métrica da matriz de confusão, e pode ser obtida conforme as extensas equações apresentadas por Congalton; Green (2008). O SCP fornece os valores de Kappa geral e por classes, bem como a matriz de confusão. Os dados foram exportados para planilhas eletrônicas, onde foram empregados os testes Z, para determinar se havia diferença significativa entre os tratamentos.

De modo a não enviesar a avaliação estatística, as amostras de validação foram coletadas antes das amostras de treinamento, e, portanto, da obtenção da classificação. O tamanho da amostra foi dimensionado de acordo com o método multinomial (CONGALTON; GREEN, 2008), adotando-se $\alpha=5 \%$, e que a classe de maior abrangência na área era mata $(30 \%$, a média dos resultados obtidos por Faria (2015), Pereira et al. (2018a) e Leite et al. (2007), para a região estudada). $O$ cálculo resultou em 740 amostras (pixels), o que 
indica que pelo menos 50 amostras para cada classe eram necessárias para que a matriz de confusão fosse preenchida corretamente. Entretanto, foram utilizados 300 pixels por classe, para garantir maior representatividade. Assim como feito para ROI's, o mesmo conjunto amostral foi fornecido para validação de todos os tratamentos. As amostras foram coletadas utilizando-se a mesma técnica descrita para coleta de ROI's, e sua distribuição foi homogênea sobre a área de estudo (Figura 1). Foram coletados, no mínimo, 18 polígonos por classe, com média de 14 pixels por polígono.

\section{RESULTADOS}

A aplicação dos classificadores utilizando-se diferentes bases de dados e estratégias amostrais produziu mapas de acurácias distintas entre si, para a maioria dos tratamentos (Tabela 3). Os valores de Kappa mostraram que a acurácia dos mapas tende a melhorar com o aumento do número de planos de informação fornecidos ao classificador (de D0 para D3) (Tabela 3). Por outro lado, a dissolução das ROI's em uma única amostra apresentou efeito significante sobre todos os tratamentos, mas de natureza diferenciada entre os classificadores: a dissolução piorou substancialmente o desempenho dos algoritmos SAM e MD, enquanto o efeito sobre o ML foi de grande melhora.

Tabela 3. Desempenho dos classificadores sobre cada base de dados e estratégia amostral. Para cada classificador, os valores de Kappa seguidos pela mesma letra maiúscula, para colunas, e minúscula, para linhas, não diferiram estatisticamente $(\mathrm{p}>0,05)$.

Table 3. Performance of the classifiers on each database and sampling design. For each classifier, Kappa values followed by the same capital letter, for columns, and lower case, for rows, did not differ statistically ( $\mathrm{p}>0.05)$.

\begin{tabular}{|c|c|c|c|c|}
\hline \multirow{2}{*}{ ROI's } & \multicolumn{4}{|c|}{ Spectral Angle Mapper (SAM) } \\
\hline & D0 & D1 & $\mathrm{D} 2$ & D3 \\
\hline Dissolvidas & $0,442 \mathrm{Aa}$ & $0,505 \mathrm{Ab}$ & $0,519 \mathrm{Ab}$ & $0,613 \mathrm{Ac}$ \\
\hline \multirow[t]{3}{*}{ Separadas } & $0,605 \mathrm{Ba}$ & $0,658 \mathrm{Bb}$ & $0,704 \mathrm{Bc}$ & $0,723 \mathrm{Bc}$ \\
\hline & \multicolumn{4}{|c|}{ Minimum Distance (MD) } \\
\hline & D0 & D1 & $\mathrm{D} 2$ & D3 \\
\hline Dissolvidas & $0,529 \mathrm{Aa}$ & $0,494 \mathrm{Ab}$ & $0,542 \mathrm{Aa}$ & $0,634 \mathrm{Ac}$ \\
\hline \multirow[t]{3}{*}{ Separadas } & $0,618 \mathrm{Ba}$ & $0,673 \mathrm{Bb}$ & $0,712 \mathrm{Bc}$ & $0,732 \mathrm{Bd}$ \\
\hline & \multicolumn{4}{|c|}{ Maximum Likelihood (ML) } \\
\hline & D0 & D1 $\ldots$ & D2 & D3 \\
\hline Dissolvidas & $0,743 \mathrm{Aa}$ & $0,806 \mathrm{Ab}$ & $0,858 \mathrm{Ac}$ & $0,849 \mathrm{Ac}$ \\
\hline Separadas & $0,665 \mathrm{Ba}$ & $-0,05^{*} \mathrm{Bb}$ & $-0,06^{*} \mathrm{Bb}$ & $-0,06 * \mathrm{Bb}$ \\
\hline
\end{tabular}

*Valores não diferiram de 0 de acordo com o teste $\mathrm{Z}(\mathrm{p}>0,05)$

O ML apresentou o melhor desempenho. Esse algoritmo foi o único a atingir valores de acurácia quase perfeita, e obteve desempenho superior aos outros classificadores em qualquer tratamento com ROI's dissolvidas, até mesmo utilizando-se a base de dados mais pobre (D0). Apesar de o ML não ter classificado a imagem de forma correta usando D1, D2 e D3 quando as ROI's não foram dissolvidas, seu desempenho ainda foi superior ao dos outros classificadores ( $p$ $<$ 0,05), quando utilizada a base de dados D0. Os classificadores MD e SAM apresentaram comportamento similar quanto à estratégia amostral e conjunto de dados, mas MD apresentou desempenho ligeiramente superior (Tabela 3).

Todos os conjuntos de dados levaram a valores de Kappa global satisfatórios, quando utilizado o ML com ROI's dissolvidas. Entretanto, apenas a o conjunto D3, que continha índices espectrais, foi capaz de elevar seguramente a acurácia de classificação a níveis satisfatórios para todas as classes mapeadas (Kappa $>0,60$ ), com efeito de melhora intensa principalmente sobre as classes "Talhões" e "Pastagem Severamente Degradada" (Figura 2). Ainda em observação à Figura 2, também é possível perceber que o aumento do número de dados utilizados tendeu a elevar os valores de índice Kappa para praticamente todas as classes mapeadas.

Cabe ressaltar que a dissolução das ROI's, apesar do efeito ambíguo sobre os classificadores, apresentou efeito muito benéfico sobre o desempenho do hardware. Enquanto o tempo demandado pela classificação com ROI's separadas foi sempre maior do que 90 minutos, a dissolução das ROI's reduziu o tempo gasto na classificação para menos do que 3 minutos, em todos os tratamentos.

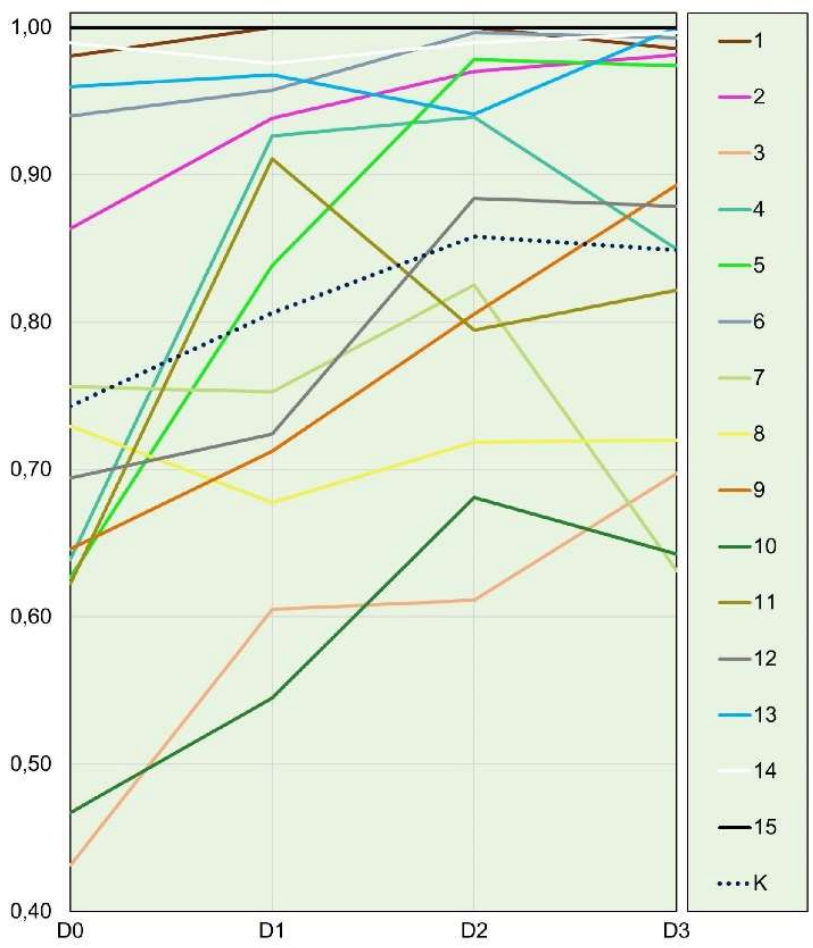

Figura 2. Valores de Kappa para o mapeamento global (K) e para cada classe mapeada (1 a 15) em função do conjunto de dados utilizado pelo classificador ML.

Figure 2. Kappa values for the global mapping $(\mathrm{K})$ and for each mapped class (1 to 15) according to the data set used by the ML classifier.

O aumento do número de planos de informação na base de dados melhorou também a qualidade visual da classificação. Mesmo para o alto desempenho do ML, a base de dados D0 deixou um aspecto fragmentário nas imagens classificadas, com pixels isolados classificados de forma incorreta. Esse efeito foi bastante atenuado pelo uso de conjuntos de dados mais robustos, como o conjunto D3, mas ainda assim foi perceptível (Figura 3).

Uma solução bastante eficaz para limpeza visual dos mapas produzidos foi a utilização do filtro Majority, do pacote de ferramentas SAGA do QGIS (Figura 3). Apesar de ser uma técnica própria para melhoria visual, o filtro remove pixels isolados, muitos deles erros de classificação. Por isso, a filtragem com raio de atuação (radius) de 3 pixels causou aumento modesto do Kappa na classificação usando ML, D0 e ROI's dissolvidos, de 0,7431 para 0,7732 ( $\mathrm{p}<0,05)$, e não alterou significativamente o Kappa para a mesma classificação quando utilizado o conjunto D3. Apenas essas duas classificações foram selecionadas para o teste de filtragem por 
conta dos resultados previamente apresentados, que indicaram ML e ROI's dissolvidos como a melhor configuração para aumentar a acurácia.

A Figura 4 apresenta o mapa final (resolução espacial de $10 \mathrm{~m}$ ) de maior concordância e qualidade visual obtidas. As classes mais abundantes foram "Matas", "Pastagens moderadamente Degradadas" e "Sem Degradação", e "Regenerações Naturais", respectivamente. A classificação apresentou acurácia quase perfeita para 12 das 15 classes, e concordância substancial para as classes "Mata", e de "Pastagens Moderadamente Degradadas" e "Sem Degradação".
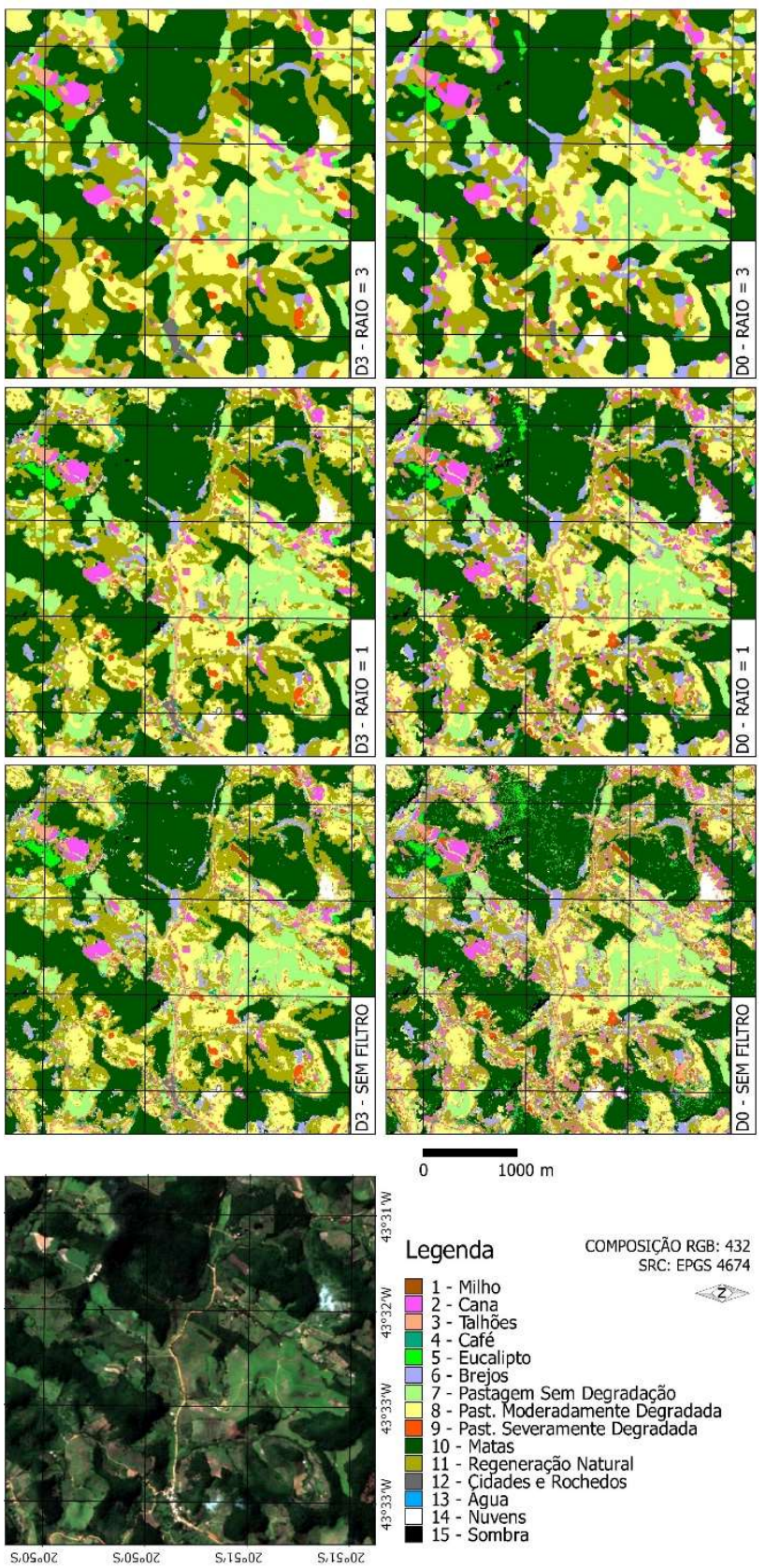

Figura 3. Comparação entre a qualidade visual das classificações oriundas de D0 e D3 usando ML e ROI's dissolvidos, antes e após uso do filtro Majority com diferentes raios de atuação.

Figure 3. Comparison between the visual quality of the classifications from D0 and D3 using ML and dissolved ROIs, before and after the use of the Majority filter with different radius of action. 4. DISCUSSÃO
Os resultados sugerem que os classificadores são muito sensíveis ao conjunto de dados e estratégia amostral adotada. A utilização de um conjunto de dados espectrais robusto (D3), que engloba dados espectrais do visível ao infravermelho próximo, bem como índices de vegetação e umidade, melhorou acentuadamente tanto a acurácia quanto a qualidade visual da classificação (Figura 3). A dissolução das ROI's em uma única amostra de treinamento otimizou o tempo de processamento, e apesar de piorar o desempenho dos classificadores MD e SAM, melhorou bastante o desempenho do ML, tornando-o superior aos outros classificadores para qualquer tratamento (Tabela 3). Além disso, ML aplicado sobre D2 ou D3 com ROI's dissolvidas, foram os únicos tratamentos capazes de elevar seguramente a acurácia da classificação a níveis adequados para todas as classes (Figura 2). O melhor desempenho do ML para mapeamentos multicategóricos já era esperado, pois diversos autores ressaltam sua superioridade aos demais classificadores testados no presente estudo (Castillejo-González et al., 2009; Yang et al., 2008; Sameen et al., 2016). Os resultados sugerem que o SCP é eficaz para mapear uso/cobertura da terra usando dados multiespectrais Sentinel 2, assim como demostraram Leroux et al. (2018).

A melhoria no desempenho do ML com a dissolução das ROI's parece estar associada à uma melhor estimativa das covariâncias para essa configuração, e à uma não estimativa da covariância para algumas classes com ROI's separadas que continham poucos pixels, conforme notificação exibida pelo próprio SCP durante a classificação. Esse erro no cálculo das covariâncias suprimiu a ocorrência de algumas classes, quando utilizadas bases de dados com muitos planos de informação, o que gerou classificações com classes monodominantes, resultando em valores de Kappa negativos (Tabela 3). ROI's com poucos pixels foram assim coletadas por restrição do tamanho diminuto das unidades de mapeamento, situação comum em mapeamentos de escala detalhada. Por isso, tornase necessário adotar a estratégia de fusão de amostras apresentada, de modo a realizar mapeamentos detalhados de forma mais acurada.

A piora no desempenho do SAM e do MD com a dissolução das ROI's parece estar relacionada à maior dispersão dos valores em torno de uma única média para cada classe, ocasionando maior confusão entre classes espectralmente próximas e com grande variabilidade intraclasse. Quando os ROI's foram fornecidos em separado, aparentemente cada vetor representou melhor a subclasse amostrada (Ex.: café jovem, café na sombra), o que diminuiu as confusões para classes de alta variabilidade espectral (por ex., Talhões e Café) (Tabela 3).

Assim, para mapeamentos multicategóricos, recomenda-se fortemente a utilização do algoritmo ML, utilizando-se ROI's dissolvidos em uma única amostra de treinamento. Para melhores resultados, indica-se a utilização do conjunto de dados multiespectrais de maior resolução espectral disponível, pois a acurácia melhorou em resposta ao aumento consecutivo de planos de informação (Tabela 3), e o tempo de processamento não consistiu em fator limitante quando as ROI's foram dissolvidas. Entretanto, caso haja interesse, a seleção de bandas com maior participação da diferenciação das classes pode ser realizada, o que diminui o volume de dados sem que haja perda de acurácia, como mostrou Faria (2015). Além disso, sugere-se a filtragem da classificação obtida 
utilizando-se o filtro Majority, para uma melhor qualidade visual sem perda de acurácia no mapa final.

Quanto ao uso da terra na região, a agricultura é praticada modestamente, e se destacam as culturas da cana, na porção nordeste, e café, na porção central. As pastagens conservadas e matas ocorrem abundantemente na escarpa da serra da Mantiqueira, devido às condições edafoclimáticas mais favoráveis e à impossibilidade de usos agrícolas mais intensivos, restringidos pelas dificuldades do manejo em altas declividades. Enquanto isso, as pastagens moderadamente degradadas dominam o planalto dissecado, devido à baixa capacidade suporte dos solos pobres em nutrientes, e do manejo inadequado frequentemente adotado. Esses resultados estão em acordo com os apresentados por Leite et al., (2007), da Rocha Júnior et al. (2017), Pereira et al. (2018a,b).

\section{CONCLUSÕES}

O Semi-automatic Classification Plugin apresenta desempenho adequado para classificação de imagens. Para melhores resultados em mapeamentos, notadamente os que assumam alta especificidade categórica e detalhamento espacial, recomenda-se fortemente que sejam adotados, simultaneamente, os seguintes procedimentos:

1) Utilização da base de dados multiespectrais com melhor resolução espectral disponível, além de índices espectrais de acordo com as classes de interesse do mapeamento;

2) Utilização do algoritmo Maximum Likelihood;

3) Coleta de amostras de treinamento abrangendo todas as variações intraclasse, com subsequente dissolução das amostras em uma única ROI.

\section{REFERÊNCIAS}

CASTILLEJO-GONZÁLEZ, I. L.; LÓPEZ-GRANADOS, F.; GARCÍA-FERRER， A.; PEÑA-BARRAGÁN， J. M.; JURADO-EXPÓSITO, M.; DE LA ORDEN, M. S.; GONZÁLEZ-AUDICANA, M. Object-and pixel-based analysis for mapping crops and their agro-environmental associated measures using QuickBird imagery. Computers and Electronics in Agriculture, New York, v. 68, n. 2, p. 207-215, 2009. DOI: https://dx.doi.org/10.1016/j.compag.2009.06.004

CONGALTON, R. G.; GREEN, K. Assessing the Accuracy of Remotely Sensed Data: Principles and Practices. 2. ed. New York: CRC Press, 2008. 183 p.

CONGEDO, L. Semi-Automatic Classification Plugin Documentation: Release 5.0.0.1. 2016. 208 p. (Technical Report). Disponível em: $<$ https://www.researchgate.net/publication/265031337_Se miAutomatic_Classification_Plugin_User_Manual>. Acesso em: $1 \overline{7}$ abr. 2017.

DA ROCHA JUNIOR, P. R.; ANDRADE, F. V.; MENDONÇA, E. S.; DONAGEMMA, G. K.; FERNANDES, R. B. A.; BHATTHARAI, R.; KALITA, P. K. Soil, water, and nutrient losses from management alternatives for degraded pasture in Brazilian Atlantic Rainforest biome. Science of The Total Environment, Amsterdam, v. 583, p. 53-63, 2017. DOI: https://dx.doi.org/10.1016/j.scitotenv.2016.12.187

DOS SANTOS, J. C.; LISBOA, G.; DE JESUS FRANÇA, L. C.; STEPKA, T. F.; DA SILVA, J. B. L.; DE MIRANDA, D. L. C.; CERQUEIRA, C. L. Relação entre variáveis meteorológicas e o uso e ocupação do solo no Sudoeste do Piauí, Brasil. Nativa, Sinop, v. 5, n. 6, p.414-420, nov./dez. 2017. DOI: http://dx.doi.org/10.5935/23187670.v05n06a06

DRUSCH, M.; DEL BELLO, U.; CARLIER, S.; COLIN, O.; FERNANDEZ, V.; GASCON, F.; HOERSCH, B.; ISOLA, C.; MARTIMORT, P.; MEYGRET, A.; SPOTO, F. SY, O. MARCHESE, F. BARGELLINI. Sentinel-2: ESA's optical high-resolution mission for GMES operational services. Remote sensing of Environment, New York, v. 120, p. 25-36, 2012. DOI: https://dx.doi.org/10.1016/j.rse.2011.11.026

EIDT, J. I.; ANDRIETTI, G.; DE FIGUEIREDO, L. F.; DEMARQUI, E.; DE ANDRADE, R. L. T.; DO AMARAL, A. G.; SCHINEIDER, R. M.; DE ALMEIDA, F. T. Monitoring metals in surface water of a small watershed in amazon region. Nativa, Sinop, v. 4, n. 4, p. 187-194, jul/ago. 2016. DOI: http://dx.doi.org/10.14583/2318-7670.v04n04a01

FARIA, M. M. Distribuição espacial do café na região das matas de Minas. 2015. 89f. Tese (Doutorado em Solos e Nutrição de Plantas) - Universidade Federal de Viçosa, Viçosa, 2015.

FOLEY, J. A.; DEFRIES. R.; ASNER, G.P.; BARFORD, C.; BONAN, G.; CARPENTER, S.T.; CHAPIN, F. S.; COE, M. T.; DAILY, G. C.; GIBBS, H. K.; HELKOWSKI, J. H.; HOLLOWAY, T.; HOWARD, E. A.; KUCHARIK, C. J.; MONFREDA, C.; PATZ, J. A.; PRENTICE, I. CO.; RAMAKUTTY, N.; SNYDER, P. K. Global consequences of land use. Science, Washington, v. 309, n. 5734, p. 570574.2005 .2 DOI: https://dx.doi.org/10.1126/science.1111772

GAO, B. C. NDWI - A normalized difference water index for remote sensing of vegetation liquid water from space. Remote sensing of environment, New York, v. 58, n. $3, \quad$ p. 257-266, 1996. DOI: https://dx.doi.org/10.1016/S0034-4257(96)00067-3

GUIMARÃES, D. P.; PIMENTA, F. M.; LANDAU, E. C. Integração Google Earth-SIG-Servidor de Mapas e o Monitoramento Ambiental. Sete Lagoas: Embrapa Milho e Sorgo, 2012. 20 p. (Circular técnica, 183)

HUETE, A.; DIDAN, K.; MIURA, T.; RODRIGUEZ, E. P.; GAO, X.; FERREIRA, L. G. Overview of the radiometric and biophysical performance of the MODIS vegetation indices. Remote sensing of environment, New York, v. 83 , n. $1-2, \quad$ p. 195-213, 2002. DOI: https://dx.doi.org/10.1016/S0034-4257(02)00096-2

JENSEN, J. R. Remote sensing of the environment: An earth resource perspective. 2. ed. Upper Saddle River: Pearson Education India, 2009. 204p.

LANDIS, J. R.; KOCH, G. G. The measurement of observer agreement for categorical data. Biometrics, Washington, v. 31, n. 1 , p. 159-174, mar. 1977. DOI: https://dx.doi.org/10.2307/2529310

LEITE, M. G. P.; FUJACO, M. A. G.; BARCELOS, E. A. S.; GONÇALVES, G. H. T.; IGREJA, F. F. Environmental analysis of small rural catchments case study: Melo CreekMG/Brazil. Management of Environmental Quality: An International Journal, v. 18, n. 6, p. 711-722, 2007. DOI: https://dx.doi.org/10.1108/14777830710826720

LEROUX, L.; CONGEDO, L.; BELLÓN, B.; GAETANO, R.; BÉGUÉ, A. Land Cover Mapping Using Sentinel-2 
Images and the Semi-Automatic Classification Plugin: A Northern Burkina Faso Case Study. In: BAGHDADI, N.; MALLET, C.; ZRIBI, M. QGIS and Applications in Agriculture and Forest. Hoboken: Wiley, 2018. 2 v. p. 119-151.

MCFEETERS, S. K. The use of the Normalized Difference Water Index (NDWI) in the delineation of open water features. International journal of remote sensing, Lisse, v. $17, \quad$ n. $7, \quad$ p. $1425-1432,1996$. DOI: https://dx.doi.org/10.1080/01431169608948714

NANNI, A.; BARROS, S. D.; DESCOVI FILHO, L.; SOUZA, M. S.; PEREIRA FILHO, N. S.; GOVEIA, S. S. QGIS User Guide - Versão 2.18. 2017. 473 p. Disponível em: $<$ https://docs.qgis.org/2.18/pdf/pt_BR/QGIS-2.18UserGuide-pt BR.pdf $>$. Acesso em: 15 dez 2017.

PEREIRA, L. F., FERREIRA, C. F. C., GUIMARÃES, R. M. F. Manejo, qualidade e dinâmica da degradação de pastagens na Mata Atlântica de Minas Gerais-Brasil. Nativa, Sinop, v. 6, n. 4, p. 370-379, jul./ago. 2018b. DOI: http://dx.doi.org/10.31413/nativa.v6i4.5542

PEREIRA, L. F.; GUIMARÃES, R. M. F.; OLIVEIRA, R. R. O. Integrando geotecnologias simples e gratuitas para avaliar usos/coberturas da terra: QGIS e Google Earth Pro. Journal of Environmental Analysis and Progress, Recife, v. 3, n. 3, p. 250-264, 2018a. DOI: http://dx.doi.org/10.24221/jeap.3.3.2018.1839.250-264

PRIETO-AMPARAN, J. A.; VILLARREAL-GUERRERO, F.; MARTINEZ-SALVADOR, M.; MANJARREZDOMÍNGUEZ, C.; SANTELLANO-ESTRADA， E.;
PINEDO-ALVAREZ, A. Atmospheric and Radiometric Correction Algorithms for the Multitemporal Assessment of Grasslands Productivity. Remote Sensing, Basel, v. 10, n. $2, \quad$ p. $219, \quad 2018$ DOI: https://dx.doi.org/10.3390/rs10020219

ROUSE, J. W.; HAAS, R. H.; SCHELL, J. A.; DEERING, D. W. Monitoring vegetation systems in the Great Plains with ERTS. Third ERTS Symposium, NASA SP-351 I, 309317, 1973

SAMEEN, M. I.; NAHHAS, F. H.; BURAIHI, F. H.; PRADHAN, B.; SHARIFF, A. R. B. M. A refined classification approach by integrating Landsat Operational Land Imager (OLI) and RADARSAT-2 imagery for landuse and land-cover mapping in a tropical area. International Journal of Remote Sensing, Basingstoke, v. 37, n. 10, p. $2358-2375,2016$. DOI: https://dx.doi.org/10.1080/01431161.2016.1176273

XIAOQIN, W.; MIAOMIAO, W.; SHAOQIANG, W.; YUNDONG, W. Extraction of vegetation information from visible unmanned aerial vehicle images. Transactions of the Chinese Society of Agricultural Engineering, v. 31, n. 5, p. 152-159, 2015. DOI: $\quad$ https://dx.doi.org/10.3969/j.issn.10026819.2015.05.022

YANG, C.; EVERITT, J. H.; MURDEN, D. Evaluating high resolution SPOT 5 satellite imagery for crop identification. Computers and Electronics in Agriculture, New York, v. 75, n. 2, p. 347-354, 2011. DOI: https://dx.doi.org/10.1016/j.compag.2010.12.012
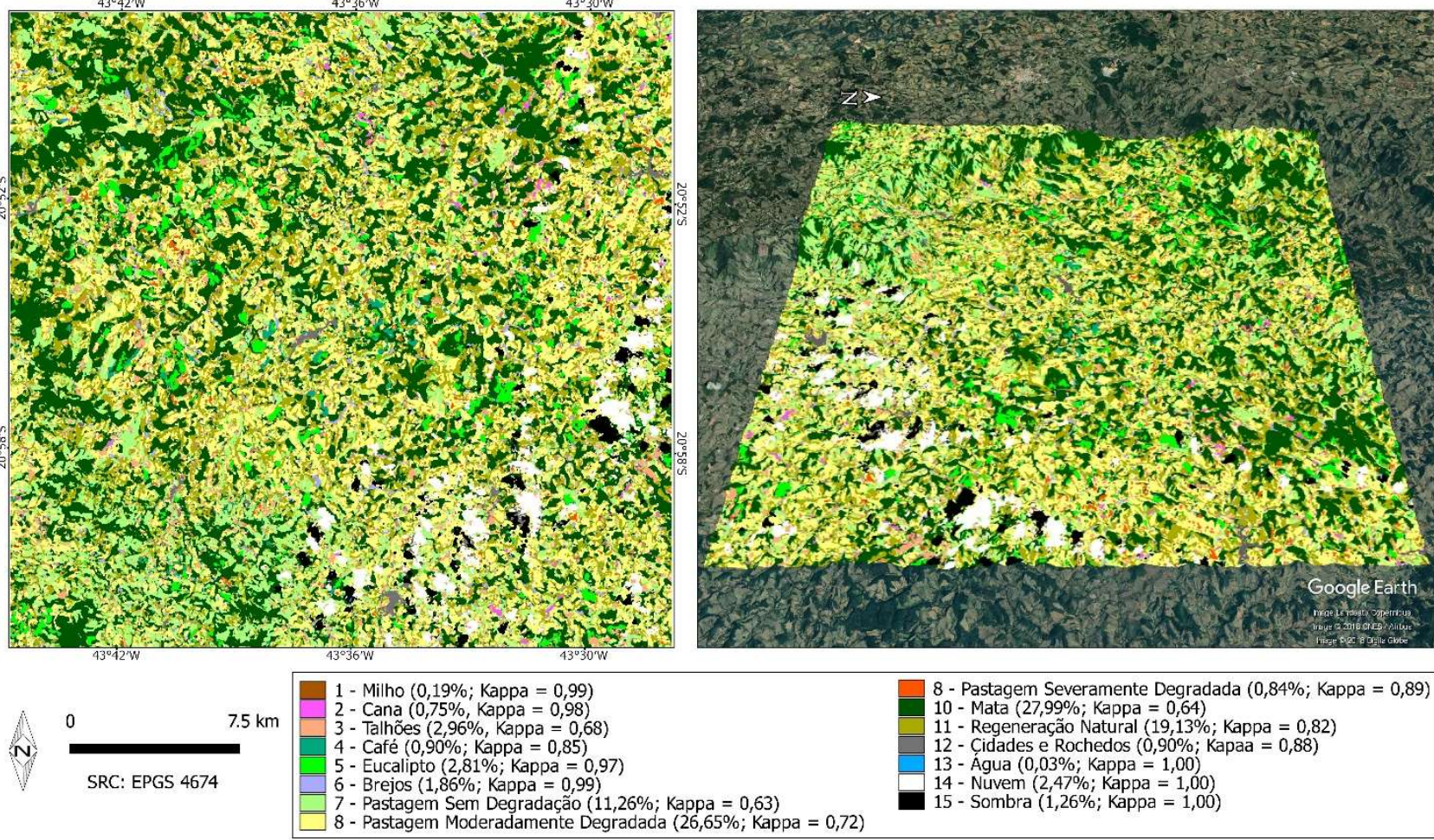

(0,84\%; Kappa $=0,89)$ 10 - Mata $(27,99 \% ;$ Kappa $=0,64)$

11 - Regeneração Natural $(19,13 \%$; Kappa $=0,82)$

12 - Çidades e Rochedos ( $0,90 \%$; Kapaa $=0,88$ )

13 - Agua $(0,03 \%$; Kappa $=1,00)$

15 - Sombra $(1,26 \%$; Kappa $=1,00)$

Pastagem Sem Degradação $(11,26 \% ;$ Kappa $=0,63)$
Pastagem Moderadamente Degradada $(26,65 \% ;$ Kappa $=0,72)$

Figura 4. À figura à esquerda apresenta o mapa de uso da terra para o local de estudo, utilizando-se ML com D3 e Majority com raio = 3. À direita, a figura mostra uma representação tridimensional sobre modelo do Google Earth Pro, com destaque para pastagens conservadas sobre as escapas da Mantiqueira, e pastagens moderadamente degradadas sobre o "mar de morros" do alto rio Doce.

Figure 4. The figure on the left presents the land use map for the study site, using ML with D3 and Majority with radius $=3$. On the right, the figure shows a three-dimensional representation on the Google Earth Pro model, with emphasis on conserved pastures on the escarpments of the Mantiqueira, and moderately degraded pastures on the hills of the upper Doce river. 